\title{
Attenuation of liver stiffness in sorafenib-treated patients with advanced hepatocellular carcinoma
}

\author{
Chien-Fu Hung ${ }^{1 *}$, Dong Liu ${ }^{2 *}$, Tsung-Han Wu ${ }^{3}$, Chao-Wei Lee ${ }^{3}$, Kuang-Tse Pan ${ }^{1}$, Ching-Ting Wang ${ }^{4}$, Hsiu-Ying Chai ${ }^{4}$, \\ Ming-Chin $\mathrm{Yu}^{2,3}, \mathrm{Yi}-\mathrm{Cheng} \mathrm{Chen}^{5}$

\begin{abstract}
'Department of Radiology, Chang Gung Memorial Hospital and Chang Gung University College of Medicine, Linkou Medical Center, Taoyuan 333, Taiwan. ${ }^{2}$ Department of Surgery, Xiamen Chang Gung Hospital, Xiamen 361000, Fujian, China.

${ }^{3}$ Department of Surgery, Chang Gung Memorial Hospital and Chang Gung University College of Medicine, Linkou Medical Center, Taoyuan 333, Taiwan. ${ }^{4}$ Department of Nuring, Chang Gung Memorial Hospital and Chang Gung University College of Medicine, Taoyuan 333, Taiwan.

${ }^{5}$ Department of Gastroenterology and Hepatology, Chang Gung Memorial Hospital and Chang Gung University College of Medicine, Taoyuan 333, Taiwan.

*Authors contributed equally to this study.
\end{abstract}

Correspondence to: Dr. Ming-Chin Yu, Department of Surgery, Chang Gung University and Memorial Hospital, No 5, Fu Hsing Street, Kuei Shan, Taoyuan 333, Taiwan. E-mail: mingchin2000@gmail.com

How to cite this article: Hung CF, Liu D, Wu TH, Lee CW, Pan KT, Wang CT, Chai HY, Yu MC, Chen YC. Attenuation of liver stiffness in sorafenibtreated patients with advanced hepatocellular carcinoma. Hepatoma Res 2017;3:52-7.

Article history:

Received: 21-11-2016

Accepted: 08-02-2017

Published: 24-03-2017

Key words:

Sorafenib,

liver stiffness,

attenuation,

advanced hepatocellular carcinoma

\section{INTRODUCTION}

Liver fibrosis is a wound-healing response and a common consequence of hepatic inflammation/injury caused by a variety of etiologies, such as infection, drugs, metabolic disorders or immune attack. ${ }^{[1]}$

\begin{abstract}
Aim: Sorafenib is a multi-tyrosine kinase inhibitor and the standard therapy for advanced hepatocellular carcinoma (HCC). This retrospective study aimed to observe the anti-fibrotic effect of sorafenib in patients with advanced HCC. Methods: Seventeen patients with advanced HCC were recruited. Shear wave velocity (SWV) using acoustic radiation force impulse elastography and non-invasive serum markers for liver fibrosis, such as the aspartate aminotransferase (AST) to alanine aminotransferase ratio (AAR), the AST to platelet ratio index, the fibrosis-4 index and the Lok index, were recorded at the beginning of sorafenib treatment and 3-6 months after sorafenib treatment in 2014-2015. Results: Nine (52.9\%) patients achieved disease control status and 8 had progressive disease after a mean duration of 11.1 months with sorafenib treatment. The mean SWV decreased from $2.37 \mathrm{~m} / \mathrm{s}$ at the beginning to $1.90 \mathrm{~m} / \mathrm{s}$ after sorafenib treatment $(P<0.01)$. This trend was observed in patients with and without liver cirrhosis (from 2.49 to $2.06 \mathrm{~m} / \mathrm{s}, P=0.06$, and from 2.32 to $1.69 \mathrm{~m} / \mathrm{s}$, $P<0.05$, respectively). Among the non-invasive serum markers, no statistically significant differences were observed except for the AAR in the cirrhotic group. Conclusion: Sorafenib has potential antif-ibrotic effects in patients with advanced HCC.
\end{abstract}

Platelet-derived growth factor (PDGF) and vascular endothelial growth factor (VEGF) are important for the sustained activation and proliferation of hepatic stellate cells (HSCs), which are activated and transformed into myofibroblasts during liver injury. ${ }^{[2,3]}$ The treatment of liver fibrosis by curing/controlling underlying liver

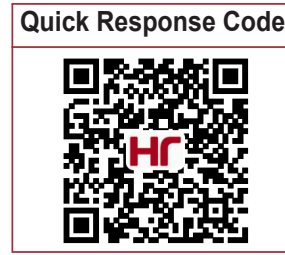


diseases or interfering with receptor/ligand interactions has been reported in clinical trials or observational studies. ${ }^{[4]}$

The inhibition of tyrosine kinase receptors for proliferative cytokines, such as PDGF, VEGF and fibroblast growth factors (FGF), could reverse liver fibrosis. The binding of PDGF to PDGF receptor (PDGFR)-b activates Ras and sequentially propagates the stimulatory signal via the phosphorylation of the mitogen-activated protein kinase (MAPK)/ extracellular-signal-regulated kinase (ERK) pathway, ${ }^{[5]}$ which regulates protein synthesis, transcription of profibrogenic genes, proliferation, cell cycle control and apoptosis in HSCs. ${ }^{[6]}$ The anti-fibrotic effect of imatinib, which occurs via the targeting of PDGF, has been observed in mouse and rat studies. ${ }^{[7]}$ Sorafenib is a multi-tyrosine kinase inhibitor that targets the receptor tyrosine kinases VEGF receptor (VEGFR) and PDGFR-b and inhibits the activation of Raf/ERK signaling pathways ${ }^{[8]}$ Sorafenib is the standard therapy for the treatment of advanced hepatocellular carcinoma (HCC) ${ }^{[9,10]}$ Recent studies have shown that sorafenib can induce anti-fibrotic effects by reducing HSC proliferation and enhancing apoptosis. ${ }^{[6,11]}$ Sorafenib also attenuates liver fibrosis and injury through the up-regulation of signal transducer and activator of transcription 3 (STAT3) phosphorylation in hepatocytes or through STAT3 inhibition in HSCs. ${ }^{[12,13]}$

Liver biopsy has been considered to be a "gold standard" for the assessment of liver fibrosis. ${ }^{[14]}$ However, a number of well-known characteristics, such as the associated risk of morbidity, including the risk of bleeding and perforation, inter-observer variability in the interpretation of biopsies, sampling variability in the context of accurate staging, monetary costs and the turnover time for results, limit the clinical application of liver biopsy. ${ }^{[15]}$ Non-invasive methods that use serum biologic markers or elastography via ultrasound and magnetic resonance imaging-based techniques have emerged recently for the indirect assessment of liver fibrosis. Acoustic radiation force impulse (ARFI) elastography is an ultrasound-based technique for quantifying the mechanical properties of tissue stiffness. ${ }^{[16,17]}$ ARFI has been utilized in comparison with various stages of liver fibrosis and shows good diagnostic accuracy in predicting hepatic fibrosis. ${ }^{[18,19]}$

We conducted an observational case-series study to assess liver fibrosis/stiffness using ARFI elastography among sorafenib-treated patients with advanced HCC to explore anti-fibrotic effects and the correlation with non-invasive methods.

\section{METHODS}

\section{Patients}

Patients with $\mathrm{HCC}$ were treated for recurrence after resection or advanced HCC as stage $\mathrm{C}$ or stage IIIIV according to the Barcelona Clinical Liver Cancer staging system or the 7th edition of American Joint Committee on Cancer/Union for International Cancer Control) staging system respectively from May 2014 to July $2015 .^{[20-22]}$ A total of 17 consecutive patients with advanced HCC were recruited retrospectively for this observational study in the clinic of Chang Gung Memorial Hospital (CGMH) fourteen patients had previously undergone surgical resection and tumor recurrence developed in the follow-up period. Sorafenib was administered as salvage treatment. The remaining three patients were unresectable with typical imaging findings. The status of advanced HCC included major portal vein thrombosis $(n=5)$ and distant metastasis ( $n=12,5$ in the lung, 2 in the bone, 2 in the peritoneum, 1 in the bone and peritoneum, 1 in a lymph node and 1 in the adrenal gland). The treatment of HCC was based on clinical practice guidelines, ${ }^{[20,21]}$ and all patients were under the care of the liver cancer team of the Linkou branch of CGMH. The daily oral dosage of sorafenib was administered and adjusted with toxicity evaluation and without drug interruption in the observation period. The dosage was deescalated with toxicity intolerance from $800 \mathrm{mg}$ to $400 \mathrm{mg}$.

This study was approved by the Institutional Review Board (IRB) of CGMH, Linkou branch (IRB No. 103-1747B). All methods of data collection were performed in accordance with the relevant guidelines and regulations of IRB in CGMH.

\section{ARFI elastography measurements}

The ARFI elastography examinations were performed with an Acuson S2000 ultrasound (Siemens Medical Solutions, Mountain View, California, USA) with ARFI technology equipment, a curvilinear array transducer operating at $4 \mathrm{MHz}(4 \mathrm{C} 1)$ and the virtual touch tissue quantification system every 3 months. With the liver parenchyma free of visible hepatic tumors, blood vessels and bile ducts, as confirmed by conventional ultrasonic images, 10 valid measurements of shear wave velocity (SWV, $\mathrm{m} / \mathrm{s}$ ) were made by a single experienced examiner (Chen YC) with the patients holding their breath for a few seconds. The results of ARFI elastography were expressed as the median of the 10 SWV measurements in the liver parenchyma. The SWV measurements in this study were validated using the ratio of the interquartile range (IQR) to the median value, which is currently used to assess the 
validity of transient elastography. An IQR/median ratio of less than 0.3 is considered to indicate a homogeneous set of measurements. ${ }^{[23,24]}$

\section{Non-invasive serologic indexes: AAR, APRI, FIB-4 index, and Lok index}

The non-invasive serologic index values at the beginning of sorafenib treatment and 3 months post-treatment were compared. The aspartate aminotransferase (AST) to alanine aminotransferase $(A L T)$ ratio (AAR), the $A S T$ to platelet ratio index (APRI), the fibrosis-4 index (FIB-4), and the Lok index for the non-invasive assessment of liver fibrosis were examined at 3 months interval. ${ }^{[25-28]}$ The variables of AST, ALT, international normalized ratio and platelets were recorded at the time of ARFI elastography.

\section{Treatment response of $\mathrm{HCC}$}

The treatment response of HCC to sorafenib was assessed based on the modified response evaluation criteria in solid tumors every 3-6 months after sorafenib administration and the treatment protocol was continued if the treatment response was disease control, including complete response (CR), partial response (PR), and stable disease (SD). ${ }^{[29]}$ Four types of response are defined as: (1) CR, which indicates the disappearance of any intratumoral arterial enhancement in all target lesions; (2) PR, which indicates a decrease of at least $30 \%$ in the sum of the diameters of viable (enhancement in the arterial phase) target lesions, taking as a reference the baseline sum of the diameters of the target lesions; (3) SD, which includes any cases that do not qualify for either PR or progressive disease (PD); and (4) PD, which indicates an increase of at least $20 \%$ in the sum of the diameters of viable (enhancing) target lesions, taking as reference the smallest sum of the diameters of viable (enhancing) target lesions recorded since treatment started. All patients have regular assessment every 2 or 3 months as 1 cycle and sorafenib administration was discontinued if the treatment response of PD was identified by liver CT assessment or if the patient's clinical condition deteriorated. All patients survived in the observation peroid; 1 lost to follow-up in 1 year; and the others were disease free or shifted to second line treatment. We used Common Terminology Criteria for Adverse Events (CTCAE) version 4.0 for adverse events severity evaluation and there was no grade IV adverse event in this study.

\section{Statistical analysis}

Clinical data were evaluated with descriptive statistics. The SWV measurements of the liver parenchyma were expressed as the median for each patient. For the overall values, the values of SWV measurements were expressed as the mean \pm standard deviation. A paired $t$-test was performed for a paired comparison of variables before and 3-6 months after treatment with sorafenib, including SWV measurements and non-invasive serologic indices. A $P$ value $<0.05$ was considered to indicate a significant difference. Statistical analysis was performed using the Statistical Package for the Social Sciences (version 19.0, IBM SPSS Statistics, New York, USA).

\section{RESULTS}

The baseline clinical characteristics of the 17 patients with advanced $\mathrm{HCC}$ are shown in Table 1. The mean age was $59.7 \pm 10.2$ years, and $14(82.4 \%)$ patients were males. Ten $(58.8 \%)$ patients had chronic hepatitis B infection, 5 (29.4\%) patients had chronic hepatitis C infection and $10(58.8 \%)$ patients had liver cirrhosis. The mean duration of sorafenib treatment was 11.1 months, and the mean survival time was 15.1 months (range: 8.3-19.1 months; $95 \%$ confidence interval: 13.317.0 months) [Table 1].

The clinical characteristics of the case series are shown in Table 2. Two patients achieved CR, 1 patient achieved PR, 6 patients experienced SD and 8 patients experienced PD after a mean of 2.5 cycles of sorafenib treatment (range 1-4 cycles). The disease control rate, including $C R, P R$, and $S D$ in the first 2 cycles was $52.9 \%$. A 64 -year-old male patient (No. 11) suffered from recurrence of mesentery HCC after the initial surgical resection and achieved a CR after 4 months of sorafenib treatment. A 34-year-old female patient (No. 12) experienced lung metastases 6 weeks after partial hepatectomy. She had a complete

Table 1: Baseline clinical characteristics

\begin{tabular}{lc}
\hline Characteristics & Data \\
\hline Patient No. & 17 \\
Age, years & $59.7 \pm 10.2$ \\
Male, \% & $14(82.4)$ \\
Liver cirrhosis, \% & $10(58.8)$ \\
HBV/HCV/none & $10 / 5 / 2$ \\
Albumin, g/dL & $4.1 \pm 0.4$ \\
AST, U/L & $56.2 \pm 37.3$ \\
ALT, U/L & $44.6 \pm 24.5$ \\
Bilirubin, $\mathrm{mg} / \mathrm{dL}$ & $0.8 \pm 0.3$ \\
INR & $1.13 \pm 0.08$ \\
Leukocytes, $10^{3} / \mathrm{L}$ & $6,288.0 \pm 2,198.0$ \\
Hemoglobin, $\mathrm{mg} / \mathrm{dL}$ & $13.5 \pm 1.7$ \\
Platelets, $10^{9} / \mathrm{L}$ & $171.5 \pm 57.2$ \\
Duration of sorafenib, months & $8.5 \pm 3.9$ \\
Survival time, months & $11.1 \pm 4.0$ \\
\hline
\end{tabular}

HBV: hepatitis B virus; HCV: hepatitis C virus; AST: aspartate transaminase; ALT: alanine transaminase; INR: international normalized ratio 
Table 2: The clinical characteristics of 17 advanced HCC patients with sorafenib treatment

\begin{tabular}{|c|c|c|c|c|c|c|c|c|c|c|c|c|c|c|}
\hline \multirow[t]{2}{*}{ No. } & \multirow{2}{*}{$\begin{array}{l}\text { Age, } \\
\text { years }\end{array}$} & \multirow{2}{*}{ Gender } & \multirow{2}{*}{$\begin{array}{l}\text { Personal } \\
\text { history }\end{array}$} & \multirow[t]{2}{*}{ HBV } & \multirow[t]{2}{*}{$\mathrm{HCV}$} & \multirow[t]{2}{*}{ Cirrhosis } & \multirow{2}{*}{$\begin{array}{c}\text { Distant } \\
\text { metastasis }\end{array}$} & \multicolumn{2}{|c|}{$\begin{array}{l}\text { ARFI elastography, } \\
\text { median, m/s }\end{array}$} & \multirow{2}{*}{$\begin{array}{c}\text { Dosage } \\
(\mathrm{mg})\end{array}$} & \multirow{2}{*}{ Staging } & \multirow{2}{*}{ Outcome } & \multirow{2}{*}{$\begin{array}{c}\text { Treatment } \\
\text { cycles }\end{array}$} & \multirow{2}{*}{$\begin{array}{c}\text { Survival } \\
\text { time } \\
\text { (months) }\end{array}$} \\
\hline & & & & & & & & Before & After & & & & & \\
\hline 1 & 59 & Male & Nil & + & - & Yes & Lung & 3.45 & 2.75 & 400 & IV & PD & 1 & 14.6 \\
\hline 2 & 67 & Male & Nil & - & + & No & Lung & 2.38 & 3.44 & 400 & IV & $\mathrm{SD} \rightarrow \mathrm{PD}$ & 2 & 17.6 \\
\hline 3 & 75 & Male & $\begin{array}{l}\text { Peptic ulcer, } \\
\text { chronic lung } \\
\text { disease }\end{array}$ & + & - & Yes & Bone & 2.98 & 1.88 & 400 & IV & PD & 2 & 14.3 \\
\hline 4 & 68 & Male & DM, HTN & - & - & No & $\begin{array}{c}\text { Bone, } \\
\text { peritoneum }\end{array}$ & 2.99 & 1.23 & 400 & IV & PD & 2 & 10.0 \\
\hline 5 & 50 & Male & Nil & + & - & No & Lung & 2.86 & 1.78 & 600 & IV & PD & 3 & 17.2 \\
\hline 6 & 61 & Female & HTN & + & - & Yes & Lung & 1.00 & 1.06 & 400 & IV & PD & 2 & 11.2 \\
\hline 7 & 61 & Male & DM, HTN & + & - & Yes & PVT & 2.68 & 2.69 & 600 & III & PD & 3 & 9.0 \\
\hline 8 & 52 & Male & Nil & - & + & No & PVT & 2.42 & 1.93 & 600 & III & SD & 3 & 13.1 \\
\hline 9 & 68 & Female & Nil & - & + & Yes & PVT & 1.29 & 2.03 & 400 & III & SD & 4 & 19.1 \\
\hline 10 & 54 & Male & Nil & + & - & Yes & PVT & 3.54 & 3.64 & 600 & III & PD & 3 & 13.1 \\
\hline 11 & 64 & Male & Peptic ulcer, HTN & + & - & Yes & Peritoneum & 2.65 & 1.42 & 600 & IV & $\mathrm{CR}^{*}$ & 3 & 12.3 \\
\hline 12 & 34 & Female & Nil & - & - & No & Lung & 1.09 & 0.90 & 800 & IV & $\mathrm{CR}^{*}$ & 4 & 13.6 \\
\hline 13 & 56 & Male & Peptic ulcer, HTN & + & - & No & Lymph node & 1.29 & 1.42 & 600 & IV & $\mathrm{SD}$ & 3 & 11.6 \\
\hline 14 & 70 & Male & DM, HTN & + & - & Yes & Peritoneum & 1.35 & 1.69 & 600 & III & $\mathrm{SD}$ & 2 & 9.9 \\
\hline 15 & 46 & Male & HTN & + & - & No & Bone & 1.37 & 1.70 & 600 & IV & SD & 2 & 9.5 \\
\hline 16 & 69 & Male & $\begin{array}{l}\text { DM, HTN, chronic } \\
\text { lung disease }\end{array}$ & - & + & Yes & $\begin{array}{l}\text { Adrenal } \\
\text { gland }\end{array}$ & 2.37 & 1.71 & 600 & IV & $\mathrm{SD}$ & 2 & 8.3 \\
\hline 17 & 61 & Male & Nil & - & + & Yes & PVT & 2.68 & 1.71 & 600 & III & PR & 2 & 8.9 \\
\hline
\end{tabular}

${ }^{*}$ One case with post-operative mesentery recurrence had another surgery for resection after 4 months of sorafenib treatment. The other case experienced multiple lung metastases after partial hepatectomy and had a complete pathologic response for lung metastases after sorafenib treatment. These pulmonary lesions enlarged initially and regressed thereafter. HBV: hepatitis B virus; HCV: hepatitis C virus; ARFI: acoustic radiation force impulse; DM: diabetes mellitus; HTN: hypertension; PVT: partial response; PD: progressive disease; SD: stable disease; CR: complete response; PR: partial response

pathological response after 9 months of sorafenib treatment. Both patients also had decreased SWV (liver stiffness) during sorafenib treatment, as indicated by ARFI elastography [Table 2]. Of the 9 patients with decreased liver stiffness, all of the reductions of SWV by ARFI elastography were $>10 \%$ from baseline, whereas there was no statistical difference in the change in SWV after sorafenib treatment between patients with and without a treatment response (decreased SWV in 5 and 4 patients with and without a treatment response, respectively, $P=1.000$ ).

The paired comparison of SWV, the AAR, the APRI, the FIB-4, and the Lok index between the beginning of sorafenib treatment and the end of treatment with sorafenib is shown in Table 3. The mean SWV was 2.37 $\pm 0.83 \mathrm{~m} / \mathrm{s}$ at the beginning of sorafenib treatment, which decreased to $1.90 \pm 0.64 \mathrm{~m} / \mathrm{s} 3$ months after sorafenib treatment $(P<0.01)$. However, there were no statistically significant differences in the noninvasive serum markers of AAR (1.39 vs. $1.15, P=$ $0.05)$, APRI (1.14 vs. 1.31, $P=0.52)$, FIB-4 (3.50 vs. $3.65, P=0.77)$, and the Lok index (0.63 vs. $0.41, P=$ 0.30 ) between the beginning of sorafenib treatment and the end of treatment [Table 3]. The decline of the mean SWV was also significant (2.32 vs. $1.69 \mathrm{~m} / \mathrm{s}$, $P<0.05)$, whereas the differences in the AAR, APRI, FIB-4 and the Lok index were not significant in the
7 patients without cirrhosis. Among the 10 patients with cirrhosis, the mean AAR decreased significantly after sorafenib treatment (1.61 vs. $1.19, P=0.04)$. The observed differences in the mean SWV by ARFI elastography, the APRI, the FIB-4 and the Lok index were not statistically significant.

\section{DISCUSSION}

To our knowledge, this investigation is the first study to evaluate the anti-fibrotic effect of sorafenib based on changes in liver parenchymal stiffness using ARFI elastography. The results of the present study showed significantly reduced stiffness of the liver parenchyma based on the SWV after short-term sorafenib treatment (reduction from 2.42 to $1.91 \mathrm{~m} / \mathrm{s}$ in 3-6 months, $P<0.01$ ), and this trend was observed in both cirrhotic and non-cirrhotic patients [Table 3].

In addition to its clinical application in advanced HCC treatment due to its ability to inhibit tumor-cell proliferation and tumor angiogenesis, ${ }^{[9,10]}$ sorafenib has been demonstrated to have anti-fibrotic effects in vivo and in vitro. ${ }^{[6,11,13]}$ These anti-fibrotic effects have been reported to occur through the inhibition of the Raf/ERK signaling pathway, which reduces HSC proliferation and enhances apoptosis. ${ }^{[6,8,11,13]}$ As observed in the present study, the decline of the 
Table 3: Comparison of SWV, AAR, APRI, FIB-4 and the Lok index at the beginning of sorafenib treatment and 3 to 6 months after sorafenib treatment

\begin{tabular}{lccccccccc}
\hline \multirow{2}{*}{ Group } & \multicolumn{2}{c}{ Overall $(\boldsymbol{n}=\mathbf{1 7})$} & \multicolumn{2}{c}{ Cirrhosis $(\boldsymbol{n}=\mathbf{1 0})$} & \multicolumn{3}{c}{ Non-cirrhosis $(\boldsymbol{n}=7)$} \\
\cline { 2 - 10 } & Beginning & After & $\boldsymbol{P}$ & Beginning & After & $\boldsymbol{P}$ & Beginning & After & $\boldsymbol{P}$ \\
SWV & $2.42 \pm 0.78$ & $1.91 \pm 0.64$ & $<0.01$ & $2.49 \pm 0.76$ & $2.06 \pm 0.73$ & 0.06 & $2.32 \pm 0.87$ & $1.69 \pm 0.45$ & $<0.05$ \\
AAR & $1.39 \pm 0.64$ & $1.15 \pm 0.36$ & 0.05 & $1.61 \pm 0.73$ & $1.19 \pm 0.39$ & 0.04 & $1.10 \pm 0.34$ & $1.10 \pm 0.36$ & 0.96 \\
APRI & $1.14 \pm 1.05$ & $1.31 \pm 0.51$ & 0.52 & $1.07 \pm 1.02$ & $1.48 \pm 0.53$ & 0.25 & $1.24 \pm 1.16$ & $1.08 \pm 0.39$ & 0.70 \\
FIB-4 & $3.50 \pm 2.45$ & $3.65 \pm 1.28$ & 0.77 & $3.89 \pm 2.29$ & $4.38 \pm 0.86$ & 0.52 & $2.94 \pm 2.74$ & $2.62 \pm 1.05$ & 0.69 \\
Lok & $0.63 \pm 1.27$ & $0.41 \pm 0.77$ & 0.30 & $1.16 \pm 1.31$ & $0.67 \pm 0.65$ & 0.49 & $-0.13 \pm 0.78$ & $0.05 \pm 0.83$ & 0.38 \\
\hline
\end{tabular}

SWV: shear wave velocity, $\mathrm{m} / \mathrm{sec}$; AAR: aspartate/alanine aminotransferase ratio; APRI: aspartate aminotransferase-platelet ratio index; FIB-4: fibrosis-4 index; Lok: Lok index

mean SWV was suggestive of the attenuation of liver parenchymal stiffness after sorafenib treatment.

Although liver biopsy is a well-known method for assessing liver fibrosis, it is not possible to perform repeated liver biopsy for the assessment of liver fibrosis in patients with advanced HCC because of possible complications and ethical issues. The development of non-invasive methods based on serum markers offers an alternative approach for clinical practice. These markers are classified as direct markers that reflect the pathophysiology of liver fibrogenesis and represent components of the extracellular matrix; indirect markers use routine laboratory data and reflect the consequences of liver damage. ${ }^{[15,25]}$ However, liver biochemistry and platelet counts could change over time in patients with deteriorating advanced HCC, and non-invasive serum markers, such as the AAR, the APRI, the FIB-4 or the Lok index, may be inadequate for assessing liver fibrosis in such patients.

With the advantage of combination with conventional B-mode ultrasound, ARFI technology can be easily used for the evaluation of liver parenchyma free of hepatic tumors, blood vessels and bile ducts, which cannot be achieved by transient elastography $\left(\right.$ Fibroscan ${ }^{\circledR}$ ). Therefore, ARFI elastography may be the better choice among non-invasive methods for evaluating the severity and serial changes of liver fibrosis during sorafenib treatment.

There are some limitations in the present study. First, this study is a case-series study, and the number of patients is small. However, the stiffness of the liver parenchyma was observed to decrease, as indicated by a reduced SWV, after sorafenib treatment. Furthermore, the results of the present study have provided a basis for future prospective large-scale studies that test the anti-fibrotic efficacy of sorafenib in the liver parenchyma. Second, the follow-up duration may be too short to see long-term changes in SWV or the stiffness of the liver parenchyma during sorafenib treatment. Third, no control group was included for comparison. Nevertheless, this study can be viewed as a pilot study to explore the anti-fibrotic effect of sorafenib in patients with advanced HCC.

In conclusion, sorafenib has potential anti-fibrotic effects and efficacy in patients with advanced HCC. Large-scale, long-term, and randomized control studies are needed to confirm the results of this study.

\section{Authors' contributions}

Study design and ARFI evaluation: M.C. Yu, Y.C. Chen Data collection and manuscript writing: C.F. Hung, D. Liu Clinical practice for HCC patients as the guideline at this institute: T.H. Wu, C.W. Lee, K.T. Pan, C.T. Wang, H.Y. Chai

\section{Financial support and sponsorship}

This study was supported by the Chang Gung Medical Foundation (CMRPG3D0511-3).

\section{Conflicts of interest}

There are no conflicts of interest.

\section{Patient consent}

Each patient was informed of the study and gave their consent.

\section{Ethics approval}

This study was approved by the Institutional Review Board (IRB) of CGMH, Linkou branch (IRB No. 1031747B).

\section{REFERENCES}

1. Friedman SL. Mechanisms of hepatic fibrogenesis. Gastroenterology 2008;134:1655-69.

2. Moreno M, Bataller R. Cytokines and renin-angiotensin system signaling in hepatic fibrosis. Clin Liver Dis 2008;12:825-52.

3. Borkham-Kamphorst E, Kovalenko E, van Roeyen CR, Gassler N, Bomble M, Ostendorf T, Floege J, Gressner AM, Weiskirchen R. Platelet-derived growth factor isoform expression in carbon tetrachloride-induced chronic liver injury. Lab Invest 2008;88:1090100 . 
4. Lee YA, Wallace MC, Friedman SL. Pathobiology of liver fibrosis: a translational success story. Gut 2015;64:830-41.

5. Pinzani M, Marra F. Cytokine receptors and signaling in hepatic stellate cells. Semin Liver Dis 2001;21:397-416.

6. Wang Y, Gao J, Zhang D, Zhang J, Ma J, Jiang H. New insights into the antifibrotic effects of sorafenib on hepatic stellate cells and liver fibrosis. J Hepatol 2010;53:132-44.

7. Kuo WL, Yu MC, Lee JF, Tsai CN, Chen TC, Chen MF. Imatinib mesylate improves liver regeneration and attenuates liver fibrogenesis in CCL4-treated mice. J Gastrointest Surg 2012;16:361-9.

8. Wilhelm SM, Adnane L, Newell P, Villanueva A, Llovet JM, Lynch M. Preclinical overview of sorafenib, a multikinase inhibitor that targets both Raf and VEGF and PDGF receptor tyrosine kinase signaling. Mol Cancer Ther 2008;7:3129-40.

9. Llovet JM, Ricci S, Mazzaferro V, Hilgard P, Gane E, Blanc JF, de Oliveira AC, Santoro A, Raoul JL, Forner A, Schwartz M, Porta C, Zeuzem S, Bolondi L, Greten TF, Galle PR, Seitz JF, Borbath I, Häussinger D, Giannaris T, Shan M, Moscovici M, Voliotis D, Bruix J; SHARP Investigators Study Group. Sorafenib in advanced hepatocellular carcinoma. N Engl J Med 2008;359:378-90.

10. Cheng AL, Kang YK, Chen Z, Tsao CJ, Qin S, Kim JS, Luo R, Feng J, Ye S, Yang TS, Xu J, Sun Y, Liang H, Liu J, Wang J, Tak WY, Pan H, Burock K, Zou J, Voliotis D, Guan Z. Efficacy and safety of sorafenib in patients in the Asia-Pacific region with advanced hepatocellular carcinoma: a phase III randomised, double-blind, placebo-controlled trial. Lancet Oncol 2009;10:25-34.

11. Hong F, Chou H, Fiel MI, Friedman SL. Antifibrotic activity of sorafenib in experimental hepatic fibrosis: refinement of inhibitory targets, dosing, and window of efficacy in vivo. Dig Dis Sci 2013;58:257-64.

12. Deng YR, Ma HD, Tsuneyama K, Yang W, Wang YH, Lu FT, Liu CH, Liu P, He XS, Diehl AM, Gershwin ME, Lian ZX. STAT3mediated attenuation of CCl4-induced mouse liver fibrosis by the protein kinase inhibitor sorafenib. J Autoimmun 2013;46:25-34.

13. Su TH, Shiau CW, Jao P, Liu CH, Liu CJ, Tai WT, Jeng YM, Yang HC, Tseng TC, Huang HP, Cheng HR, Chen PJ, Chen KF, Kao $\mathrm{JH}$, Chen DS. Sorafenib and its derivative SC-1 exhibit antifibrotic effects through signal transducer and activator of transcription 3 inhibition. Proc Natl Acad Sci U S A 2015;112:7243-8.

14. Rockey DC, Caldwell SH, Goodman ZD, Nelson RC, Smith AD; American Association for the Study of Liver Diseases. Liver biopsy. Hepatology 2009;49:1017-44.

15. Asrani SK. Incorporation of noninvasive measures of liver fibrosis into clinical practice: diagnosis and prognosis. Clin Gastroenterol Hepatol 2015;13:2190-204.

16. Nightingale K, Soo MS, Nightingale R, Trahey G. Acoustic radiation force impulse imaging: in vivo demonstration of clinical feasibility. Ultrasound Med Biol 2002;28:227-35.

17. Fahey BJ, Nightingale KR, Nelson RC, Palmeri ML, Trahey GE. Acoustic radiation force impulse imaging of the abdomen: demonstration of feasibility and utility. Ultrasound Med Biol 2005;31:1185-98.

18. Friedrich-Rust M, Nierhoff J, Lupsor M, Sporea I, FierbinteanuBraticevici C, Strobel D, Takahashi H, Yoneda M, Suda T, Zeuzem $\mathrm{S}$, Herrmann E. Performance of Acoustic Radiation Force Impulse imaging for the staging of liver fibrosis: a pooled meta-analysis. $J$ Viral Hepat 2012;19:e212-9.

19. Nierhoff J, Chávez Ortiz AA, Herrmann E, Zeuzem S, Friedrich-Rust M. The efficiency of acoustic radiation force impulse imaging for the staging of liver fibrosis: a meta-analysis. Eur Radiol 2013;23:304053.

20. European Association For The Study Of The Liver; European Organisation For Research And Treatment Of Cancer. EASLEORTC clinical practice guidelines: management of hepatocellular carcinoma. J Hepatol 2012;56:908-43.

21. Omata M, Lesmana LA, Tateishi R, Chen PJ, Lin SM, Yoshida H, Kudo M, Lee JM, Choi BI, Poon RT, Shiina S, Cheng AL, Jia JD, Obi S, Han KH, Jafri W, Chow P, Lim SG, Chawla YK, Budihusodo U, Gani RA, Lesmana CR, Putranto TA, Liaw YF, Sarin SK. Asian Pacific Association for the Study of the Liver consensus recommendations on hepatocellular carcinoma. Hepatol Int 2010;4:439-74.

22. American Joint Committee on Cancer (AJCC). Cancer Staging Manual. 7th ed. New York: Springer; 2009.

23. Frulio N, Laumonier H, Carteret T, Laurent C, Maire F, Balabaud C, Bioulac-Sage P, Trillaud H. Evaluation of liver tumors using acoustic radiation force impulse elastography and correlation with histologic data. J Ultrasound Med 2013;32:121-30.

24. Fraquelli M, Rigamonti C, Casazza G, Conte D, Donato MF, Ronchi G, Colombo M. Reproducibility of transient elastography in the evaluation of liver fibrosis in patients with chronic liver disease. Gut 2007;56:968-73.

25. Papastergiou V, Tsochatzis E, Burroughs AK. Non-invasive assessment of liver fibrosis. Ann Gastroenterol 2012;25:218-31.

26. Lok AS, Ghany MG, Goodman ZD, Wright EC, Everson GT, Sterling RK, Everhart JE, Lindsay KL, Bonkovsky HL, Di Bisceglie AW, Lee WM, Morgan TR, Dienstag JL, Morishima C. Predicting cirrhosis in patients with hepatitis $\mathrm{C}$ based on standard laboratory tests: results of the HALT-C cohort. Hepatology 2005;42:282-92.

27. Lin ZH, Xin YN, Dong QJ, Wang Q, Jiang XJ, Zhan SH, Sun Y, Xuan SY. Performance of the aspartate aminotransferase-to-platelet ratio index for the staging of hepatitis C-related fibrosis: an updated meta-analysis. Hepatology 2011;53:726-36.

28. Xiao G, Yang J, Yan L. Comparison of diagnostic accuracy of aspartate aminotransferase to platelet ratio index and fibrosis-4 index for detecting liver fibrosis in adult patients with chronic hepatitis B virus infection: a systemic review and meta-analysis. Hepatology 2015;61:292-302.

29. Lencioni R, Llovet JM. Modified RECIST (mRECIST) assessment for hepatocellular carcinoma. Semin Liver Dis 2010;30:52-60. 